\title{
ONE-DIMENSIONAL LIE FOLIATIONS WITH GENERIC SINGULARITIES IN COMPLEX DIMENSION THREE
}

\author{
ALBETÃ MAFRA and BRUNO SCARDUA ${ }^{\bowtie}$ \\ (Received 17 April 2010; accepted 25 October 2010) \\ Communicated by M. K. Murray
}

\begin{abstract}
We prove that a germ of a one-dimensional holomorphic foliation with a generic singularity in dimension two or three that exhibits a Lie group transverse structure in the complement of some codimension one analytic subset is logarithmic, that is, given by a system of closed meromorphic one-forms with simple poles. In the global context, we prove that a foliation by curves in a three-dimensional complex manifold with generic singularities and a Lie group transverse structure off a codimension one analytic subset is logarithmic; that is, it is given by a system of closed meromorphic one-forms with simple poles.
\end{abstract}

2010 Mathematics subject classification: primary 57R30; secondary 22E15.

Keywords and phrases: holomorphic foliation with singularities, Lie transverse structure.

\section{Introduction}

Foliations are an important tool in the study of the topology of manifolds, dynamics and the theory of singularities. In the theory of foliations it is very useful to consider the transverse structure. Among the simplest transverse structures are Lie group transverse structures, homogeneous transverse structures and Riemannian transverse structures.

Here we consider foliations with a Lie group transverse structure. Roughly speaking, this means that the foliation is given by an atlas of submersions taking values in a given Lie group $G$ and with transition maps given by restrictions of left translations on the group $G$. This atlas is called the transverse model for $\mathcal{F}$. We shall refer to such a foliation as a $G$-foliation. The theory of $G$-foliations is a well developed subject and follows the original work of Blumenthal [2].

In codimension one, the Riemann-Koebe uniformization theorem implies that any holomorphic $G$-foliation is transversely additive (that is, its transverse model is the additive group of complex numbers) and is therefore given by a closed holomorphic

(c) 2011 Australian Mathematical Publishing Association Inc. 1446-7887/2011 \$16.00 
one-form (see [16]). Using this fact, together with some extension techniques from the theory of holomorphic foliations, it is possible to study in detail codimension one holomorphic foliations on complex projective spaces that admit a Lie group transverse structure on the complement of some invariant algebraic subset (see [16, 17]). In the nontrivial case, this invariant algebraic subset has codimension one.

On the other hand, as in the local situation, it is possible to prove that, under some mild conditions, an isolated singularity of a holomorphic one-form in two dimensions that admits a $G$-transverse structure on the complement of its set of local separatrices is given by a closed meromorphic one-form (see also Theorem 9). In particular, in the irreducible case, the singularity is analytically equivalent to its formal normal form as introduced in $[13,14]$.

Our aim is to motivate the study of the case where the codimension is at least two, beginning with the very simplest case. More precisely, we study one-dimensional holomorphic foliations on complex manifolds with generic singularities that admit a Lie group transverse structure on the complement of some analytic invariant subset of codimension one.

In codimension one, a basic example of a class of foliations with generic singularities that have a Lie group transverse structure on the complement of some analytic hypersurface is the class of logarithmic foliations. A logarithmic foliation on a complex manifold $V$ is one given by a closed meromorphic one-form $\eta$ with simple poles. A Darboux foliation on $V$ is a logarithmic foliation given by a meromorphic one-form $\eta$ as follows:

$$
\eta=\sum_{j=1}^{r} \lambda_{j} \frac{d f_{j}}{f_{j}}
$$

where the functions $f_{j}$ are meromorphic on $V$ and $\lambda_{j} \in \mathbb{C} \backslash\{0\}$ (see $[4,17]$ for more on logarithmic and Darboux foliations). It is well known that any logarithmic one-form $\eta$ in the complex projective space $\mathbb{C} P^{m}$ is of Darboux type (see [16]).

By a logarithmic foliation of dimension one on a complex manifold $V$ of dimension $m$, we mean a foliation that is given by a system of $m-1$ closed meromorphic oneforms $\eta_{1}, \ldots, \eta_{m-1}$, all of which have simple poles and are linearly independent in the complement of their sets of poles. A Darboux foliation of dimension one is a logarithmic foliation given by one-forms $\eta_{j}$ of Darboux type. An isolated singularity of a holomorphic vector field $X$ is said to be without resonances if the eigenvalues of the linear part $D X$ at the singular point are linearly independent over $\mathbb{Q}$. Local linearization of such a (nonresonant) singularity is assured if this singularity is in the Poincaré domain; that is, the convex hull of its eigenvalues does not contain the origin. In the other case, that is, in the Siegel domain, there are Diophantine conditions that assure local linearization (see [3, 19]).

Our results below are a first step in the understanding of the possible Lie group and homogeneous transverse structures (see [9]) for holomorphic foliations with singularities. The description of the local situation with a generic singularity is better stated in terms of germs as follows. 
Theorem 1. Let $\mathcal{F}$ be a one-dimensional holomorphic foliation defined in an open connected neighborhood $V$ of the origin $(0,0,0) \in \mathbb{C}^{3}$ with a linearizable singularity without resonances at the origin. Assume that $\mathcal{F}$ has a $G$-transverse structure outside an invariant codimension one analytic subset $\Lambda$ of $V$. If each irreducible component of $\Lambda$ contains the origin, then $\mathcal{F}$ is logarithmic.

As in the global case, we have the following result.

THeorem 2. Let $\mathcal{F}$ be a one-dimensional holomorphic foliation with singularities on a three-dimensional connected complex manifold $V$ and a $G$-transverse structure in the complement of a codimension one analytic subset $\Lambda$ of $V$. Suppose that each irreducible component of $\Lambda$ contains a singular point of $\mathcal{F}$ that is linearizable without resonances. Then $\mathcal{F}$ is logarithmic.

We point out that the restriction to three dimensions (that is, $\operatorname{codim} \mathcal{F}=2$ ) is just for notational simplicity. The main conclusion of the paper, that the presence of generic singularities forces the transverse structure to be Abelian, holds for one-dimensional foliations in three or more dimensions, as one can mimic the proof of Lemma 12 in higher dimensions.

\section{Lie foliations and differential forms}

We begin with a definition.

Definition 3. Let $\mathcal{F}$ be a foliation of a manifold $V$ of codimension $\ell$. Given an $\ell$ dimensional Lie group $G$, we say that $\mathcal{F}$ admits a Lie group transverse structure with model $G$, or a $G$-transverse structure for short, if there is an open cover $V=\bigcup_{j \in J} U_{j}$ of $V$ such that the following conditions are met.

(i) On each open set $U_{j}$, there is a submersion $f_{j}: U_{j} \rightarrow G$ such that the leaves of $\left.\mathcal{F}\right|_{U_{j}}$ are the level sets of $f_{j}$.

(ii) on each nonempty intersection $U_{i} \cap U_{j} \neq \emptyset$, there is a locally constant map $g_{i j}: U_{i} \cap U_{j} \rightarrow G$ such that $f_{i}=g_{i j} f_{j}$.

In other words, $\mathcal{F}$ is defined by submersions $f_{j}: U_{j} \rightarrow G$ that differ on $U_{i} \cap U_{j}$ by left translations $f_{i}=L_{g_{i j}}\left(f_{j}\right)$ where the elements $g_{i j} \in G$ are locally constant. When these conditions hold, we call $\mathcal{F}$ a $G$-foliation or simply a Lie foliation.

The next result characterizes $G$-foliations.

Let $G$ be a Lie group and $\left\{\omega_{1}, \ldots, \omega_{\ell}\right\}$ be a basis of the Lie algebra of $G$. Then there is a family of constants $c_{i j}^{k}$, called the structure constants of the Lie algebra in the given basis, such that

$$
d \omega_{k}=\sum_{i<j} c_{i j}^{k} \omega_{i} \wedge \omega_{j} .
$$

Theorem 4 (Darboux-Lie [9]). Let $\mathcal{F}$ be a codimension $\ell$ foliation on $V$ and let $G$ be a Lie group of dimension $\ell$. Let $\left\{\omega_{1}, \ldots, \omega_{\ell}\right\}$ be a basis for the Lie algebra of $G$ with 
structure constants $c_{i j}^{k}$. If $\mathcal{F}$ admits a G-transverse structure, then there are one-forms $\Omega_{1}, \ldots, \Omega_{\ell}$ in $V$ satisfying the following conditions.

(i) $\left\{\Omega_{1}, \ldots, \Omega_{\ell}\right\}$ is a rank $\ell$ integrable system that defines $\mathcal{F}$.

(ii) $d \Omega_{k}=\sum_{i<j} c_{i j}^{k} \Omega_{i} \wedge \Omega_{j}$.

If $\mathcal{F}, V$ and $G$ are complex and holomorphic, then the $\Omega_{j}$ may be taken to be holomorphic.

Conversely, given a maximal rank system of one-forms $\Omega_{1}, \ldots, \Omega_{\ell}$ in $V$ such that

$$
d \Omega_{k}=\sum_{i, j}^{k} c_{i j}^{k} \Omega_{i} \wedge \Omega_{j},
$$

where the $c_{i j}^{k}$ are the structure constants of a given basis $\left\{\omega_{1}, \ldots, \omega_{\ell}\right\}$ of the Lie algebra of $G$, the following conditions are satisfied.

(iii) For each point $p \in V$, there are a neighborhood $U_{p}$ of $p$ in $V$ and a submersion $f_{p}: U_{p} \rightarrow G$ that defines $\mathcal{F}$ in $U_{p}$ such that $f_{p}^{*}\left(\omega_{j}\right)=\Omega_{j}$ in $U_{p}$ for all $j \in$ $\{1, \ldots, \ell\}$.

(iv) If $V$ is simply connected, then we may take $U_{p}$ to be $V$.

(v) If $U_{p} \cap U_{q} \neq \emptyset$, then there is a locally constant element $g_{p q}$ of $G$ such that $f_{q}=L_{g_{p q}}\left(f_{p}\right)$ in $U_{p} \cap U_{q}$.

In particular, $\mathcal{F}$ has a $G$-transverse structure.

\section{Examples}

The most trivial example of a $G$-foliation is given by the product foliation on a manifold $V=G \times N$, that is, the product of a Lie group $G$ with a manifold $N$. The leaves of the foliation are of the form $\{g\} \times N$ where $g \in G$. Next we give a type of nonsingular example based on an algebraic construction.

ExAmple 5. Let $H$ be a closed (normal) subgroup of a Lie group $G$. We consider the action $\Phi: H \times G \rightarrow G$ given by $\Phi(h, g)=h g$ and the quotient map $\pi: G \rightarrow G / H$ (a fibration) that defines a foliation $\mathcal{F}$ on $G$.

Given $x \in \mathcal{F}_{g}=\pi^{-1}(H g)$, we have $\pi(x)=H g$ and $\Phi_{h}(x)=h x$. However, the fact that

$$
\pi\left(\Phi_{h}(x)\right)=\pi(h x)=H h x=H x
$$

implies that

$$
\Phi_{h}(x) \in \pi^{-1}(H x)=\mathcal{F}_{x}
$$

and the orbit $O(g)=H g$ is transverse to the fiber $\pi^{-1}(H g)$. Hence $\mathcal{F}$ is a foliation that is invariant under the transverse action $\Phi$.

Now let $G$ be a simply connected group, let $H$ be a discrete subgroup of $G$ and let $F: H \rightarrow \operatorname{Diff}(G)$ be the natural representation given by $F(h)=L_{h}$. The universal covering of $G / H$ is $G$, with the projection $\pi: G \rightarrow G / H$ and $\pi_{1}(G / H) \simeq H$, 
because $\pi \circ f(g)=H f(g)=H g$ for $f \in \operatorname{Aut}(G)$. Thus if $f(g) \simeq g$, then $f(g) g^{-1} \in H$ and $f(g)=h g$ for some unique $h \in H$. Therefore $f=L_{h}$ and we may define an isomorphism $f \mapsto h$ from $\operatorname{Aut}(G)$ to $H$. Thus we may write $F: \pi_{1}(G / H) \rightarrow \operatorname{Diff}(G)$ and $\Phi: \pi_{1}(G / H) \times G \rightarrow G$. The map $\Psi: H \times G \times G \rightarrow G \times G$ given by $\Psi\left(h, g_{1}, g_{2}\right)=$ $\left(L_{h}\left(g_{1}\right), L_{h}\left(g_{2}\right)\right)$ is a properly discontinuous action and defines a quotient manifold $V=(G \times G) / \Psi$ whose equivalence classes are the orbits of $\Psi$. We have the following facts.

(i) There exists a fibration $\sigma: V \rightarrow G / H$ with fiber $G$ induced by $\pi: G \rightarrow G / H$ and structure group isomorphic to $F(H) \subset \operatorname{Diff}(G)$. In fact, we define $\sigma: V \rightarrow G / H$ by $\sigma\left(\Psi\left(h, g_{1}, g_{2}\right)\right)=\pi\left(g_{1}\right)$. For every point $x \in G / H$ there is a neighborhood $U \subset G / H$ such that $\pi^{-1}(U)$ is isomorphic with $U \times H$, and thus there is an isomorphism between $\sigma^{-1}(U)$ and $U \times G$.

(ii) The natural (zero-dimensional) foliation $\mathcal{F}$ on $G$ given by the classes $\mathrm{Hg}$ for each $g \in G$ is $\Phi$-invariant. Therefore the product foliation $G \times \mathcal{F}$ on $G \times G$, which has dimension $\operatorname{dim} G$, is $\Psi$-invariant and induces a foliation $\mathcal{F}_{0}$ on $V$, which is called the suspension of $\mathcal{F}$ with respect to $\Phi$. The suspension $\mathcal{F}_{0}$ is then a foliation transverse to the projection $\sigma: V \rightarrow G / H$.

Example 6 (Euler equation). The Euler equation is the germ of a saddle node at the origin $(0,0) \in \mathbb{C}^{2}$ given by $\Omega=0$, where

$$
\Omega=x^{2} d y-(y+x) d x .
$$

Note that $d \Omega=(2 x+1) d x \wedge d y$ and $d \Omega=\eta \wedge \Omega$, where $\eta$ is the closed form

$$
2 \frac{d x}{x}+\frac{d x}{x^{2}}
$$

Since

$$
\eta=\frac{d\left(x^{2} e^{-1 / x}\right)}{x^{2} e^{-1 / x}}
$$

if we define

$$
\omega=\left(x^{2} e^{-1 / x}\right)^{-1} \Omega,
$$

then $\omega$ is a closed meromorphic one-form in the complement of the (only) separatrix, the $y$ axis. Because $\Omega \wedge \omega=0$ off this axis, we conclude that the Euler equation admits a Lie transverse structure off the set of separatrices. Nevertheless it is well known that, due to the absence of a second separatrix, the Euler equation is not analytically conjugate to its formal normal form. This needs to be considered in the statements of our results in the next section.

\section{Germs of generic singular Lie foliations in dimension two}

The generic local case in dimension two is described below. A germ $\mathcal{F}$ of a holomorphic foliation at the origin $(0,0) \in \mathbb{C}^{2}$ is called a nonresonant singularity if 
it is given in suitable local coordinates $(x, y)$ centered at $(0,0)$ by an equation of the form

$$
x(1+A(x, y)) d y-\lambda y(1+B(x, y)) d x=0,
$$

where $A$ and $B$ are holomorphic, $A(0)=B(0)=0$ and $\lambda \notin \mathbb{Q}$. In this case, the set of separatrices $\Lambda$ is the union of the $x$ and $y$ axes. The following proposition is derived from [21, Theorem II.3.1].

Proposition 7. Let $\mathcal{F}$ be a germ of a nonresonant singularity at the origin $(0,0) \in \mathbb{C}^{2}$. Suppose that $\mathcal{F}$ is a $G$-foliation in the complement of the set of separatrices $\Lambda$. Then the germ $\mathcal{F}$ is analytically linearizable. Moreover, any closed holomorphic one-form $\Omega$ defining $\mathcal{F}$ in $V \backslash \Lambda$, where $V$ is a neighborhood of the origin, extends to $V$ as a logarithmic one-form.

Proof. First we remark that we may suppose that $G=(\mathbb{C},+)$ because $\operatorname{dim} G=1$. Therefore there is a closed holomorphic one-form $\Omega$ in the complement of the separatrices such that $\Omega$ defines the foliation in this open set and the foliation is transversely additive in the complement of its local separatrices. In particular, it is transversely projective in this complement and we may apply [21, Theorem II.3.1] to conclude that the singularity is analytically linearizable. We may assume that the set of separatrices $\Lambda$ is the union of the $x$ and $y$ axes.

Now let $V$ be a neighborhood of the origin and $\Omega$ be a closed holomorphic oneform that defines $\mathcal{F}$ in $V \backslash \Lambda$. Then there are local coordinates $(\tilde{x}, \tilde{y})$ such that $\{(x, y): y=0\}=\{(\tilde{x}, \tilde{y}): \tilde{y}=0\}$ and $\{(x, y): x=0\}=\{(\tilde{x}, \tilde{y}): \tilde{x}=0\}$, and further $\mathcal{F}$ may be written in the form

$$
\tilde{x} d \tilde{y}-\lambda \tilde{y} d \tilde{x}=0 .
$$

We define the closed one-form $\tilde{\omega}$ by

$$
\tilde{\omega}=\frac{d \tilde{y}}{\tilde{y}}-\lambda \frac{d \tilde{x}}{\tilde{x}}
$$

Then $\tilde{\omega}$ is a meromorphic one-form defining $\mathcal{F}$, so we may write $\tilde{\omega}$ as $\tilde{f} \Omega$, where $\tilde{f}$ is a meromorphic function off the $\tilde{x}$ and $\tilde{y}$ axes. Expanding the Laurent series of $\tilde{f}$ with respect to the axes, we see that

$$
\tilde{f}(\tilde{x}, \tilde{y})=\sum_{i, j \in \mathbb{Z}} f_{i j} \tilde{x}^{i} \tilde{y}^{j}
$$

with coefficients $f_{i j} \in \mathbb{C}$. Since

$$
0=d \tilde{\omega}=d \tilde{f} \wedge \Omega+\tilde{f} d \Omega=d \tilde{f} \wedge \Omega,
$$

$\tilde{f}$ is a Laurent series that corresponds to a first integral of $\mathcal{F}$, that is, $d \tilde{f} \wedge \tilde{\omega}=0$. Using this Laurent series, we see that

$$
\sum_{i, j \in \mathbb{Z}}(i+\lambda j) f_{i j} \tilde{x}^{i} \tilde{y}^{j}=0
$$


and so we may conclude that $f_{i j}=0$ whenever $(i, j) \neq(0,0)$, since $\lambda \notin \mathbb{Q}$. Hence $\tilde{f}$ is the constant $f_{00}$ and therefore $\Omega=f_{00}^{-1} \tilde{\omega}$. This implies that the Laurent series of the one-form $\tilde{x} \tilde{y} \Omega$ around the $\tilde{x}$ and $\tilde{y}$ axes contains only positive powers, and so it converges to a holomorphic one-form. Therefore $\Omega$ extends to the separatrices as a meromorphic one-form with simple poles.

Now we consider a more general situation than that examined in Proposition 7. Let $\mathcal{F}$ be a holomorphic foliation in a neighborhood $U$ of the origin with an isolated singularity at the origin. Since we are concerned with the behavior of $\mathcal{F}$ in a neighborhood of the origin, we may assume that $\operatorname{sing}(\mathcal{F})=\{(0,0)\}$. We resolve singularities for $\mathcal{F}$ at $(0,0)$ (see $[6,15,18]$ ) to obtain a complex surface $\widetilde{U}$ and a proper holomorphic map $\pi: \widetilde{U} \rightarrow U$ that is a finite composition of quadratic blow ups such that the exceptional divisor $D=\pi^{-1}(0)$ is a normal crossing without triple points. We note also that $D$ is a finite union $D=\bigcup_{j=1}^{m} \mathbb{P}_{j}$, where the $\mathbb{P}_{j}$ are projective lines with negative self-intersection in $\widetilde{U}$. The pull back foliation $\widetilde{\mathcal{F}}=\pi^{*}(\mathcal{F})$ is a foliation with singularity set $\operatorname{sing}(\widetilde{\mathcal{F}})$ in $D$, consisting of irreducible isolated singularities that are either nondegenerate singularities or saddle node singularities. Nondegenerate singularities satisfy equations of the form

$$
x(1+A(x, y)) d y-\lambda y(1+B(x, y)) d x=0,
$$

where $A$ and $B$ are holomorphic, $A(0)=B(0)=0$ and $\lambda \notin \mathbb{Q}_{+}$. These singularities are said to be resonant if $\lambda \in \mathbb{Q}_{-}$and nonresonant otherwise. Saddle node singularities satisfy equations of the form

$$
y^{t+1} d x-\left[x\left(1+\lambda y^{t}\right)+A(x, y)\right] d y=0,
$$

where $t$ is a positive integer, $A$ is a holomorphic function of order at least $t+2$ at $(0,0)$ and $\lambda \in \mathbb{C}$. The strong separatrix of the saddle node is the $x$ axis. If the singularity admits another separatrix, then it is necessarily smooth, transverse to the strong separatrix and it can be taken as the other coordinate axis. This will be called the central manifold of the saddle node.

Any component $\mathbb{P}_{j}$ of $D$ is either $\tilde{\mathcal{F}}$-invariant or everywhere transverse to $\widetilde{\mathcal{F}}$. In the latter case, we say that $\mathbb{P}_{j}$ is a dicritical component.

The following definition generalizes the above notion of a nonresonant singularity.

Definition 8 (Nonresonant germ). A germ of a holomorphic foliation $\mathcal{F}$ at the origin $(0,0) \in \mathbb{C}^{2}$ is called nonresonant if the following conditions are met.

(i) The resolution of singularities of $\mathcal{F}$ at the origin exhibits no saddle node.

(ii) Each connected component of the invariant part of the exceptional divisor $\pi^{-1}(0)$ contains a nonresonant (nondegenerate) singularity.

Given an analytic invariant subset $\Lambda$ of $U$ such that each irreducible component of $\Lambda$ contains the origin, we may write $\Lambda$ as the union of irreducible components $\bigcup_{j=1}^{r} \Lambda_{j}$. 
The strict transform $\tilde{\Lambda}$ of $\Lambda$ may then be defined by

$$
\widetilde{\Lambda}=\overline{\pi^{-1}(\Lambda \backslash\{(0,0)\})}=\bigcup_{j=1}^{r} \widetilde{\Lambda}_{j},
$$

where $\widetilde{\Lambda}_{j}=\overline{\pi^{-1}\left(\Lambda_{j} \backslash\{(0,0)\}\right)}$; it is a union of $\tilde{\mathcal{F}}$-invariant irreducible components in $\widetilde{U}$.

A component $\Lambda_{j}$ of $\Lambda$ will be called dicritical if its strict transform $\tilde{\Lambda}_{j}$ contains no singularity; that is, if it is a leaf of the foliation $\tilde{\mathcal{F}}$. We write $\operatorname{Dic}(\Lambda)$ for the union of dicritical components of $\Lambda$. The exceptional divisor $D$ is $\pi^{-1}(0,0)$. The large transform of $\Lambda$ is by definition the union of the strict transform of $\Lambda$ and the exceptional divisor, that is, $\pi^{-1}(\Lambda)=\tilde{\Lambda} \cup D$.

Now we are in a position to prove our main result in two dimensions.

THEOREM 9. Let $\mathcal{F}$ be a nonresonant germ of a holomorphic foliation at the origin $(0,0) \in \mathbb{C}^{2}$. Suppose that $\mathcal{F}$ is a $G$-foliation outside of a germ of an analytic subset $\Lambda$ of codimension one. Then $\mathcal{F}$ is given by a closed meromorphic one-form $\Omega$ in some open subset $U^{*}$ of the form $U \backslash \operatorname{Dic}(\Lambda)$. In the nondicritical case, $U^{*}=U$.

In order to prove Theorem 9 we shall need the following extension lemma in addition to Proposition 7.

Lemma 10. Let $\mathcal{F}$ be a germ of a nondegenerate singularity at the origin $(0,0) \in \mathbb{C}^{2}$. Suppose that there is a closed meromorphic one-form $\Omega$ defining $\mathcal{F}$ in a neighborhood of the singularity minus one of the local separatrices. Then the germ of the singularity $\mathcal{F}$ is analytically conjugate to its formal normal form. In particular, $\Omega$ extends meromorphically to the other separatrix.

Proof. First we prove that the formal normal form of the singularity converges. In suitable local coordinates $(x, y)$ in some neighborhood $U$ of the origin, the set of separatrices is the union of the $x$ and $y$ axes. We may assume that $\Omega$ is defined on $U \backslash\{(x, y): x=0\}$ for some neighborhood $U$ of the origin. Since $\Omega$ is closed, its polar set is invariant under the foliation $\mathcal{F}=\mathcal{F}(\Omega)$, and therefore the polar set of $\Omega$ in $U \backslash\{(x, y): x=0\}$ is the $y$-axis.

Let $\Lambda$ denote the separatrix $\{(x, y): y=0\}$. Let $a=\operatorname{Res}_{\Lambda}(\Omega) \in \mathbb{C}$ be the residue of $\Omega$ at $\Lambda$ and let the positive integer $k$ be the order of $\Lambda$ as an irreducible component of the polar set of $\Omega$. If we take $U$ to be a bidisc, then we can write

$$
\Omega(x, y)=a \frac{d y}{y}+d \frac{\varphi}{y^{k}},
$$

where $\varphi$ is a holomorphic function in $U \backslash\{(x, y): x=0\}$. If $q \in \Lambda \backslash\{(0,0)\}$, then there are local coordinates $(\tilde{x}, \tilde{y})$ in a neighborhood $U_{q}$ of $q$ such that $\tilde{x}(q)=\tilde{y}(q)=0$, $\{(\tilde{x}, \tilde{y}): \tilde{y}=0\}=\{(x, y): y=0\} \cap U_{q}$, and

$$
\Omega(\tilde{x}, \tilde{y})=a \frac{d \tilde{y}}{\tilde{y}}+d \frac{1}{\tilde{y}^{k}} .
$$


In particular, $\tilde{y}: U_{q} \rightarrow \mathbb{C}$ is a holomorphic submersion that defines $\mathcal{F}$ in $U_{q}$. Now

$$
\Omega(\tilde{x}, \tilde{y})=\frac{a \tilde{y}^{k}-k}{\tilde{y}^{k+1}} d \tilde{y},
$$

and for each nonempty intersection $U_{q} \cap U_{q^{\prime}}$ the corresponding change of coordinates in the local submersions above leaves the vector field

$$
X(y)=\frac{y^{k+1}}{\left(a y^{k}-k\right)} \frac{\partial}{\partial y}
$$

invariant. There are therefore two possibilities for the local holonomy map $h$ of the leaf $\Lambda \backslash\{(0,0)\}=\{(x, y): y=0\} \backslash\{(0,0)\}$. If $a=0$, then $h$ leaves the vector field

$$
X(y)=-\frac{y^{k+1}}{k} \frac{\partial}{\partial y}
$$

invariant and therefore $h$ is analytically conjugate to a map of the form

$$
h(y)=\frac{\mu y}{\left(1+b y^{k}\right)^{1 / k}},
$$

which is a formal normal form for the map $h$. If $a \neq 0$, then $h$ leaves a vector field of the form

$$
X(y)=\frac{y^{k+1}}{\left(1+a y^{k}\right)} \frac{\partial}{\partial y}
$$

invariant, and since this is a holomorphic vector field in its formal normal form, we may also conclude that $h$ is analytically conjugate to its formal normal form.

Now, according to [14], because the local holonomy map $h$ is analytically conjugate to its formal normal form, the singularity $\mathcal{F}$ is analytically conjugate to its formal normal form. This proves the first part of the lemma.

We divide the remaining part of the proof into several cases. If $\lambda \notin \mathbb{R}_{-}$, then the singularity is in the Poincaré domain and because $\lambda \notin \mathbb{Q}_{+}$it is without resonances. By the Poincaré-Dulac linearization theorem [8] the singularity is analytically linearizable. Thus from now on we may assume that $\lambda \in \mathbb{R}_{-}$. We now consider each remaining case separately.

Case 1: Nonresonance. In this case $\lambda \notin \mathbb{Q}$. The formal normal form is linear and we just apply Proposition 7 to finish the proof.

Case 2: Holomorphic first integral. In this case the foliation is given in suitable coordinates by a linear one-form $\alpha=k x d y+\ell y d x$ for some positive integers $k$ and $\ell$. We may assume that $k$ and $\ell$ are coprime. Set $\omega:=1 /(x y)$ and $\alpha=k d y / y+$ $\ell d x / x$. Then $\Omega \wedge \omega=0$ in $U \backslash\{(x, y): x=0\}$ so that $\Omega=f \omega$ for some meromorphic function $f$ defined in $U \backslash\{(x, y): x=0\}$.

Since $\Omega$ and $\omega$ are closed, $d f \wedge \omega=0$ and $f$ is a meromorphic first integral for $\alpha$ in $U \backslash\{(x, y): x=0\}$. Since we have assumed that $k$ and $\ell$ are coprime, the form $\alpha$ has a 
holomorphic first integral $f_{0}(x, y)=y^{k} x^{\ell}$ with connected irreducible fibers except for $f_{0}^{-1}(0)$. This implies, by a local version of the Stein factorization theorem (see [15]), that $f=\xi\left(f_{0}\right)$ for some meromorphic function $\xi$ in a punctured neighborhood of the origin $0 \in \mathbb{C}$. Since $\Omega$ is meromorphic in $U \backslash\{(x, y): x=0\}$ and is therefore meromorphic in $\{(x, y): y=0\} \backslash\{0\}$, it follows that $f=\xi\left(y^{k} x^{\ell}\right)$ is meromorphic in $\{(x, y): y=0\} \backslash\{(0,0)\}$. Hence the function $\xi$ extends meromorphically to the origin $0 \in \mathbb{C}$. Therefore $\Omega=f \omega$ extends to $U$ as a meromorphic one-form.

Case 3: Resonant nonlinearizable. Now $\lambda \in \mathbb{Q}_{-}$, say $\lambda=-\ell / k$ for relatively prime positive integers $k$ and $\ell$. By hypothesis, the singularity is not analytically linearizable. According to [14], it has a formal model of the form

$$
\hat{\Omega}_{k / \ell, n, \mu}=\ell\left[1+(\mu-1) u^{n}\right] y d x+k\left(1+\mu u^{n}\right] x d y,
$$

where $u=x^{\ell} y^{k}$ and $\mu \in \mathbb{C}$. By the first part of our proof, the foliation is analytically conjugate to this formal normal form. Since the foliation is given by a closed oneform off the separatrix $\{(x, y): x=0\}$, we may conclude that the analytic model for the foliation is indeed of the form

$$
\hat{\Omega}_{k, \ell}=k x d y+\ell y\left[1+(\sqrt{-1} / 2 \pi) x^{\ell} y^{k}\right] d x
$$

(see [16, p. 179] or [7, Remark 7, Lemma 3]). Each normal form $\hat{\Omega}_{k, \ell}$ admits an integrating factor $\hat{h}=x^{\ell+1} y^{k+1}$. Thus there are closed rational one-forms

$$
\hat{\omega}_{k, \ell}=\frac{1}{x^{\ell+1} y^{k+1}} \quad \text { and } \quad \hat{\Omega}_{k, \ell}=d\left[-\frac{1}{\left(x^{\ell} y^{k}\right)}+\log \left(\frac{\sqrt{-1} \ell}{2 \pi}\right) x\right] .
$$

Arguing as above, we may deduce that $\Omega=\hat{f} \hat{\omega}_{k, \ell}$ for some meromorphic function $\hat{f}$ in $U \backslash\{(x, y): x=0\}$ such that $d \hat{f} \wedge \hat{\omega}_{k, \ell}=0$. Comparing poles enables us to conclude that $\hat{f}$ is indeed holomorphic off the union of the $x$ and $y$ axes $\{(x, y): x y=0\}$.

We claim that $\hat{f}$ is constant. If not, then $\hat{f}$ is a nonconstant meromorphic first integral for $\mathcal{F}$. Since the singularity is not dicritical, this implies that $\mathcal{F}$ admits a holomorphic first integral. Now, according to [15], if a resonant Siegel type singularity admits a nonconstant formal or holomorphic first integral, then it is analytically linearizable, which is a contradiction. Therefore, we conclude that $\hat{f}$ is constant (see also the proof of [17, Lemma 3]) and so $\Omega$ extends to the separatrices as a meromorphic one-form with simple poles.

Proof of Theorem 9. We choose representatives for the germs $\mathcal{F}$ and $\Lambda$ as above in a neighborhood $U$ of the origin. We also denote these representatives by $\mathcal{F}$ and $\Lambda$. The foliation $\tilde{\mathcal{F}}$ is a $G$-foliation in

$$
\pi^{-1}(U \backslash \Lambda)=\tilde{U} \backslash \pi^{-1}(\Lambda)=\tilde{U} \backslash(D \cup \tilde{\Lambda})
$$

Applying the arguments used in the proof of Proposition 7 enables us to deduce that there is a closed holomorphic one-form $\tilde{\Omega}$ in $\tilde{U} \backslash(D \cup \tilde{\Lambda})$ that defines $\tilde{\mathcal{F}}$ in this open subset. 
We now investigate the possibility of extending $\Omega$ as a meromorphic one-form to a given component $\mathbb{P}_{j}$ of the exceptional divisor and to a component $\tilde{\Lambda}_{j}$ of the strict transform $\tilde{\Lambda}$. We say that a given point $\tilde{p} \in \pi^{-1}(\Lambda)=\tilde{\Lambda} \cup D$ is regular if it is not an intersection point of two irreducible components of $\pi^{-1}(0)$. If $\mathbb{P}_{j}$ is a component of $D$, then denote by $\mathbb{P}_{j}^{*}$ its set of regular points. The set of regular points in $\tilde{\Lambda}_{j}$ is denoted by $\tilde{\Lambda}_{j}^{*}$.

Let $\mathbb{P}_{j}$ be a dicritical component of the exceptional divisor. We claim that the one-form $\tilde{\Omega}$ extends meromorphically to $\mathbb{P}_{j}^{*}$. In fact, take a regular point $\tilde{p} \in \mathbb{P}_{j}$. Because this component is dicritical, there are local coordinates $(x, y)$ centered at $\tilde{p}$ such that $\mathbb{P}_{j}$ is the $x$ axis and $\tilde{\mathcal{F}}$ is given by $d x=0$. The one-form $\tilde{\Omega}(x, y)$ is closed and meromorphic off the $x$ axis. We can write $\tilde{\Omega}(x, y)=\mu d y / y+d f(x, y)$ for some $\mu \in \mathbb{C}$ and some function $f$ meromorphic off the $x$ axis. Because $\tilde{\Omega} \wedge d x=0$, it follows that $\mu / y+\partial f / \partial y=0$. Using Laurent series, we conclude that $\mu=0$ and $f_{y}=0$, so $f$ depends only on the variable $x$. Because $f$ is meromorphic off the $x$ axis, we conclude that $f$ is meromorphic in a neighborhood of $\tilde{p}$.

We claim that the one-form $\tilde{\Omega}$ extends meromorphically to any nondicritical component $\mathbb{P}_{j_{0}}$ of the exceptional divisor. Indeed, we first observe that, by hypothesis, since $\mathbb{P}_{j_{0}}$ is invariant under $\tilde{\mathcal{F}}$ there is a path $Q_{0}, \ldots, Q_{r}$, where $Q_{v}=\mathbb{P}_{j_{v}}$ is an invariant projective line, $Q_{0}=\underset{\sim}{\mathbb{P}} j_{0}, Q_{v} \cap Q_{v+1} \neq \emptyset$, and $Q_{r}=\mathbb{P}_{j}$ contains a nonresonant singularity, say $q_{r}$, in $\operatorname{sing}(\tilde{\mathcal{F}})$. This singularity corresponds either to a corner, that is, an intersection of two invariant components of the exceptional divisor, or to some point intersection $\mathbb{P}_{j} \cap \tilde{\Lambda}_{v}$. In either case, because $\tilde{q}$ is an irreducible nondegenerate singularity, Proposition 7 applies and enables us to deduce that $\tilde{\Omega}$ extends meromorphically to a neighborhood of $\tilde{q}$.

By a classical theorem of Levi (see [20]), a meromorphic form defined in the complement of an analytic codimension one irreducible subset $\Lambda$ of a complex manifold $V$ extends meromorphically to this subset provided that it admits a meromorphic extension to some open subset that intersects $\Lambda$. Thus the one-form $\tilde{\Omega}$ extends meromorphically to all regular points in $\mathbb{P}_{j}$.

Let $\tilde{p}$ be a nonregular point in $\mathbb{P}_{j}$. If $\tilde{p}$ is a singular point of $\tilde{\mathcal{F}}$, then by the above argument, $\tilde{\Omega}$ extends meromorphically to a neighborhood of $\tilde{p}$. Thus it remains to show that $\tilde{\Omega}$ extends meromorphically to the nonregular points $\tilde{p}$ corresponding to intersections of $\mathbb{P}_{j}$ with dicritical components of the exceptional divisor, say $\mathbb{P}_{j} \cap \mathbb{P}_{v}$. Given such a nonregular point $\tilde{p}$, there are local coordinates $(x, y)$ centered at $\tilde{p}$ such that $\mathbb{P}_{j}$ corresponds to the $x$ axis, $\mathbb{P}_{v}$ to the $y$ axis, and the foliation $\tilde{\mathcal{F}}$ is given in these coordinates by $d y=0$. The one-form $\tilde{\Omega}$ is meromorphic off the $x$ and $y$ axes, and by the above arguments extends to $\mathbb{P}_{j}^{*}$ and to $\mathbb{P}_{v}^{*}$ as a meromorphic form. Therefore $\tilde{\Omega}$ is meromorphic in $U \backslash\{\tilde{p}\}$ where $U$ is some neighborhood of the point $\tilde{p}$.

Hartogs' classical extension theorem, which assures the extension of meromorphic forms through analytic subsets of codimension at least two (see [10, 12]), enables us to conclude that $\tilde{\Omega}$ extends meromorphically to $\tilde{p}$. Now we have proved that $\Omega$ extends to $\mathbb{P}_{j}=Q_{r}$. The intersection $Q_{r-1} \cap Q_{r}=\left\{q_{r}\right\}$ is a singularity and the one-form $\Omega$ is 
defined and meromorphic in a neighborhood of this singularity minus one of its two local separatrices. More precisely, $\Omega$ is defined off the separatrix contained in $Q_{r-1}$.

If $q_{r}$ admits a holomorphic first integral, then by Lemma 10 the one-form $\Omega$ extends meromorphically to a neighborhood of $q_{r}$. If $q_{r}$ does not admit a holomorphic first integral, then by Lemma 10 the one-form $\Omega$ extends meromorphically to a neighborhood of $q_{r}$. Hence, as above, the one-form extends to the regular points in $Q_{r-1}$ as a meromorphic one-form. An argument similar to that given above shows that the one-form $\Omega$ always extends to the component $Q_{r-1}$. An inductive argument then shows that $\Omega$ extends to $\mathbb{P}_{j_{0}}=Q_{0}$ as a meromorphic one-form.

Now we finish the proof. From the discussion above, we may conclude that $\tilde{\Omega}$ extends meromorphically to all invariant components of the exceptional divisor and to all components $\tilde{\Lambda}_{j}$ that intersect some invariant component of the exceptional divisor. Also, $\tilde{\Omega}$ extends to the dicritical part of the exceptional divisor except, possibly, to those branches $\tilde{\Lambda}_{i}$ that meet the exceptional divisor at dicritical components. These components form an analytic invariant subset $\tilde{\Gamma}$ of $\tilde{\Lambda}$ that projects under $\pi$ (using the proper mapping theorem from [11]) onto the analytic invariant subset $\operatorname{Dic}(\Lambda)$ of $\Lambda$. The one-form $\Omega$ is meromorphic in some neighborhood $\tilde{U}$ of $\pi^{-1}(\Lambda) \backslash \tilde{\Gamma}$ and therefore the one-form $\Omega$ is meromorphic in some open subset $U \backslash \operatorname{Dic}(\Lambda)$. Here $U$ is a neighborhood of the origin $(0,0) \in \mathbb{C}^{2}$.

Corollary 11. Let $\mathcal{F}$ be a nondicritical germ of a foliation at $0 \in \mathbb{C}^{2}$ and assume that each projective line in the resolution of 0 exhibits no saddle nodes and contains a nonresonant singularity. If there is a germ of a codimension one analytic subset $\Lambda$ at the origin such that $\mathcal{F}$ is a $G$-foliation off $\Lambda$, then $\mathcal{F}$ is a germ of a logarithmic foliation.

Proof. By the proof of Theorem 9, there is a closed meromorphic one-form $\Omega$ that defines $\mathcal{F}$ in a neighborhood of the origin. This one-form is the projection by the resolution morphism of a closed meromorphic one-form $\tilde{\Omega}$ defined in a neighborhood of the resolution divisor that has simple poles in a neighborhood of each nonresonant singularity. By our hypothesis on the singularities of the resolution divisor, $\widetilde{\Omega}$ has simple poles, and, therefore, so has $\Omega$. Hence the foliation $\mathcal{F}$ is logarithmic.

\section{Lie foliations with singularities in dimension three}

Now let $\mathcal{F}$ be a one-dimensional holomorphic foliation with isolated singularities on a complex manifold $V$. We shall say that $\mathcal{F}$ admits a $G$-transverse structure if the corresponding nonsingular foliation $\mathcal{F}_{0}:=\left.\mathcal{F}\right|_{V \backslash \operatorname{sing}(\mathcal{F})}$ admits a $G$-transverse structure.

Let $p \in \operatorname{sing}(\mathcal{F})$ be an isolated singularity. Take a small neighborhood $\Delta_{p}$ of $p$ in $V$, biholomorphic to a ball in $\mathbb{C}^{m}$, such that $\Delta_{p} \cap \operatorname{sing}(\mathcal{F})=\{p\}$, and set $\Delta_{p}^{*}=\Delta_{p} \backslash\{p\}$. Then $\Delta_{p}^{*}$ is simply connected, and, by the classical theory of transverse Lie foliations (see also Theorem 4), the restriction $\left.\mathcal{F}\right|_{\Delta_{p}^{*}}$ is given by a holomorphic submersion $P: \Delta_{p}^{*} \rightarrow G$. By Hartogs' extension theorem, the map extends to a holomorphic map $P: \Delta_{p} \rightarrow G$ that is a first integral for $\mathcal{F}$ in $\Delta_{p}$. 
Now suppose that $\mathcal{F}$ admits a $G$-transverse structure on $V \backslash \Lambda$, where $\Lambda$ is an analytic subset of $V$ of codimension at least two such that $\Lambda \cap \operatorname{sing}(\mathcal{F})=\emptyset$. Again by Hartogs' extension theorem, the one-forms $\Omega_{1}, \ldots, \Omega_{\ell}$ obtained in Theorem 4 extend as holomorphic forms to $V$. Moreover, also by Theorem 4, the $G$-transverse structure extends to $V$ as long as the system $\left\{\Omega_{1}, \ldots, \Omega_{\ell}\right\}$ has maximal rank at the points of $\Lambda$.

We claim that this is the case because $\Lambda$ does not intersect the singular set of $\mathcal{F}$. Let $q \in \Lambda$. Then since $q$ is not a singular point for $\mathcal{F}$, there is a neighborhood $U_{q}$ of $q$ in $V$ and a system of holomorphic forms $\left\{\omega_{1}, \ldots, \omega_{\ell}\right\}$, defined and of maximal rank in $U_{q}$, that defines $\mathcal{F}$ in $U_{q}$. Therefore there is a holomorphic matrix $A=\left(a_{i j}\right)_{i, j=1}^{\ell}$ whose coefficients are the holomorphic functions $a_{i j}: U_{q} \rightarrow \mathbb{C}$ such that $\Omega_{i}=\sum_{j=1}^{\ell} a_{i j} \omega_{j}$ for $j=1, \ldots, \ell$.

If the determinant $\operatorname{det}(A)$ vanishes at some point in $U_{q} \cap \Lambda$, then it vanishes in some analytic subset of codimension one that intersects $\Lambda$. Therefore the system $\left\{\Omega_{1}, \ldots, \Omega_{\ell}\right\}$ does not have maximal rank in some analytic subset of codimension one that intersects $\Lambda$, which is a contradiction. Therefore $A$ is nonsingular at the points of $U_{q} \cap \Lambda$ and consequently $\left\{\Omega_{1}, \ldots, \Omega_{\ell}\right\}$ has maximal rank at the points of $U_{q}$. Hence we shall consider the case where $\mathcal{F}$ admits a $G$-transverse structure on $V \backslash \Lambda$ and $\Lambda$ is analytic of codimension one in $V$. We shall also assume that $\Lambda$ is invariant under $\mathcal{F}$.

Lemma 12. Let $X=\sum_{j=1}^{3} \lambda_{j} x_{j} \partial / \partial x_{j}$ lie in a connected neighborhood $U$ of $0 \in \mathbb{C}^{3}$, where $\lambda_{1}, \lambda_{2}, \lambda_{3}$ are linearly independent over $\mathbb{Q}$. Let $\mathcal{F}(X)$ denote the onedimensional foliation induced by $X$. Suppose that there are holomorphic oneforms $\Omega_{1}, \Omega_{2}$ defined in $U_{0}:=U \backslash \bigcup_{j=1}^{3}\left\{\left(x_{1}, x_{2}, x_{3}\right): x_{j}=0\right\}$ such that the following conditions are satisfied.

(i) $d \Omega_{k}=c_{12}^{k} \Omega_{1} \wedge \Omega_{2}$, where the $c_{12}^{k}$ are the structure constants of a Lie algebra of a Lie group $G$ of dimension two.

(ii) The foliation $\mathcal{F}(X)$ induced by $X$ is given in $U_{0}$ by the integrable system $\left\{\Omega_{1}, \Omega_{2}\right\}$ of maximal rank.

Then $\Omega_{1}$ and $\Omega_{2}$ are closed and extend to $U$ as closed meromorphic one-forms with simple poles.

Proof. Let $a_{1}, a_{2}, a_{3}$ be complex numbers and define the closed one-form $\Theta$ by

$$
\Theta=\sum_{k=1}^{3} a_{k} d x_{k} / x_{k} .
$$

Then $\Theta(X)=0$ if and only if $\sum_{k=1}^{3} a_{k} \lambda_{k}=0$. Thus we can take one-forms $\Theta_{1}$ and $\Theta_{2}$, given by $\Theta_{j}=\sum_{j=1}^{3} a_{k}^{j} d x_{k} / x_{k}$ for $a_{k}^{j} \in \mathbb{C}$, that are linearly independent in the complement of the coordinate hyperplanes and satisfy $\Theta_{1}(X)=\Theta_{2}(X)=0$.

Once we fix such one-forms, the foliation $\mathcal{F}(X)$ is defined by the integrable system $\left\{\Theta_{1}, \Theta_{2}\right\}$ in $U$. This definition and part (ii) enable us to write

$$
\Omega_{1}=a_{1} \Theta_{1}+a_{2} \Theta_{2} \text { and } \Omega_{2}=b_{1} \Theta_{1}+b_{2} \Theta_{2}
$$


in $U_{0}$ for some holomorphic functions $a_{1}, a_{2}, b_{1}, b_{2}$ in $U_{0}$ with the property that $a_{1} b_{2}-b_{1} a_{2}$ does not vanish in $U_{0}$. Now, since $\Theta_{j}$ is closed,

$$
d \Omega_{1}=d a_{1} \wedge \Theta_{1}+d a_{2} \wedge \Theta_{2} \text { and } d \Omega_{2}=d b_{1} \wedge \Theta_{1}+d b_{2} \wedge \Theta_{2} .
$$

Thus

$$
d \Omega_{1} \wedge \Theta_{1}=\left(d a_{1} \wedge \Theta_{1}+d a_{2} \wedge \Theta_{2}\right) \wedge \Theta_{1}=d a_{2} \wedge \Theta_{2} \wedge \Theta_{1} .
$$

Since $d \Omega_{1}=c_{12}^{1} \Omega_{1} \wedge \Omega_{2}$, we see that

$$
d \Omega_{1}=c_{12}^{1}\left(a_{1} b_{2}-a_{2} b_{1}\right) \Theta_{1} \wedge \Theta_{2},
$$

and hence $d \Omega_{1} \wedge \Theta_{1}=0$. Thus we may deduce that

$$
d a_{2} \wedge \Theta_{1} \wedge \Theta_{2}=0
$$

We may also deduce that $\Theta_{1} \wedge \Theta_{2}$ is equal to

$$
\left(a_{1}^{1} a_{2}^{2}-a_{1}^{2} a_{2}^{1}\right) \frac{d x_{1} \wedge d x_{2}}{x_{1} x_{2}}+\left(a_{1}^{1} a_{3}^{2}-a_{1}^{2} a_{3}^{1}\right) \frac{d x_{1} \wedge d x_{3}}{x_{1} x_{3}}+\left(a_{2}^{1} a_{3}^{2}-a_{3}^{1} a_{2}^{2}\right) \frac{d x_{2} \wedge d x_{3}}{x_{2} x_{3}} .
$$

We write

$$
\Theta_{1} \wedge \Theta_{2}=\alpha_{12} \frac{d x_{1} \wedge d x_{2}}{x_{1} x_{2}}+\alpha_{13} \frac{d x_{1} \wedge d x_{3}}{x_{1} x_{3}}+\alpha_{23} \frac{d x_{2} \wedge d x_{3}}{x_{2} x_{3}} .
$$

Now we note that, given a holomorphic function $f\left(x_{1}, x_{2}, x_{3}\right)$ in $U_{0}$, the equation

$$
d f \wedge \Theta_{1} \wedge \Theta_{2}=\left(\frac{f_{x_{1}} \alpha_{23}}{x_{2} x_{3}}-\frac{f_{x_{2}} \alpha_{13}}{x_{1} x_{3}}+\frac{f_{x_{3}} \alpha_{12}}{x_{1} x_{2}}\right) d x_{1} \wedge d x_{2} \wedge d x_{3}
$$

is satisfied. Therefore $d f \wedge \Theta_{1} \wedge \Theta_{2}=0$ if and only if

$$
\alpha_{23} x_{1} f_{x_{1}}-\alpha_{13} x_{2} f_{x_{2}}+\alpha_{12} x_{3} f_{x_{3}}=0 .
$$

Now, expanding the Laurent series $f=\sum_{i, j, k \in \mathbb{Z}} f_{i j k} x_{1}^{i} x_{2}^{j} x_{3}^{k}$ enables us to see that the last equation is equivalent to

$$
\sum_{i, j, k \in \mathbb{Z}}\left(i \alpha_{23}-j \alpha_{13}+k \alpha_{12}\right) f_{i j k} x_{1}^{i} x_{2}^{j} x_{3}^{k}=0
$$

which is equivalent to

$$
\left(i \alpha_{23}-j \alpha_{13}+k \alpha_{12}\right) f_{i j k}=0
$$

for all $i, j, k \in \mathbb{Z}$. Recall that

$$
\alpha_{12}=a_{1}^{1} a_{2}^{2}-a_{1}^{2} a_{2}^{1}, \quad \alpha_{13}=a_{1}^{1} a_{3}^{2}-a_{1}^{2} a_{3}^{1}, \quad \alpha_{23}=a_{2}^{1} a_{3}^{2}-a_{3}^{1} a_{2}^{2}
$$

and that

$$
a_{1}^{1} \lambda_{1}+a_{2}^{1} \lambda_{2}+a_{3}^{1} \lambda_{3}=0, \quad a_{1}^{2} \lambda_{1}+a_{2}^{2} \lambda_{2}+a_{3}^{2} \lambda_{3}=0
$$


Thus the two complex vectors $\left(\alpha_{12}, \alpha_{13}, \alpha_{23}\right)$ and $\left(\lambda_{1}, \lambda_{2}, \lambda_{3}\right)$ are collinear, that is, $\alpha_{12}=t \lambda_{1}, \alpha_{13}=t \lambda_{2}$ and $\alpha_{23}=t \lambda_{3}$ for some $t \in \mathbb{C}^{*}$. Thus $d f \wedge \Theta_{1} \wedge \Theta_{2}=0$ if and only if $\left(i \lambda_{1}-j \lambda_{2}+k \lambda_{3}\right)=0$ for all $i, j, k \in \mathbb{Z}$. By the nonresonance hypothesis, the only solution to the last equation is trivial. Therefore a holomorphic function $f\left(x_{1}, x_{2}, x_{3}\right)$ in $U_{0}$ satisfies $d f \wedge \Theta_{1} \wedge \Theta_{2}=0$ if and only if $f$ is constant.

The above argument shows that $a_{2}$ is constant. Similarly $a_{1}, b_{1}$ and $b_{2}$ are constant in $U_{0}$ and

$$
\left(\begin{array}{l}
\Omega_{1} \\
\Omega_{2}
\end{array}\right)=C\left(\begin{array}{l}
\Theta_{1} \\
\Theta_{2}
\end{array}\right)
$$

for some nonsingular $2 \times 2$ complex matrix $C$. Since $\Theta_{1}$ and $\Theta_{2}$ are logarithmic oneforms, this proves that $\Omega_{1}$ and $\Omega_{2}$ extend to $U$ as closed meromorphic one-forms with simple poles.

Proof Theorem 2. By Theorem 4, there are holomorphic one-forms $\Omega_{1}$ and $\Omega_{2}$ in $V_{0}:=V \backslash \Lambda$ such that $d \Omega_{k}=\sum_{i<j} c_{i j}^{k} \Omega_{i} \wedge \Omega_{j}$, where the $c_{i j}^{k}$ are the structure constants of a Lie algebra of the Lie group $G$. The foliation $\mathcal{F}$ is given in $V_{0}$ by the integrable system $\left\{\Omega_{1}, \Omega_{2}\right\}$ of maximal rank.

Let $\Lambda_{v}$ be an irreducible component of $\Lambda$. By hypothesis, there is an analytically linearizable singularity $q_{v} \in \Lambda_{v} \cap \operatorname{sing}(\mathcal{F})$ without resonances. By Lemma 12 , each $\Omega_{j}$ extends to a neighborhood of $q_{v}$ in $V$ as a closed meromorphic one-form with simple poles. The extension theorem of Levi asserts that each $\Omega_{j}$ extends to $\Lambda_{v}$ as a closed meromorphic one-form. Therefore $\mathcal{F}$ is logarithmic.

Theorem 1 follows immediately from Theorem 2.

\section{Acknowledgement}

We are very much indebted to the referee for very constructive comments, careful reading, and valuable suggestions and various hints that have greatly improved this article.

\section{References}

[1] A. Behague and B. Scárdua, 'Foliations invariant under Lie group transverse actions', Monatsh. Math. 153 (2008), 295-308.

[2] R. Blumenthal, 'Transversely homogeneous foliations', Ann. Inst. Fourier (Grenoble) 29 (1979), 143-158.

[3] A. D. Brjuno, 'Analytical form of differential equations', Trans. Moscow Math. Soc. 25 (1971), $131-288$.

[4] O. Calvo Andrade, 'Irreducible components of the space of holomorphic foliations', Math. Ann. 299 (1994), 751-767.

[5] C. Camacho and A. Lins Neto, Geometric Theory of Foliations (Birkhäuser, Berlin, 1985).

[6] C. Camacho and P. Sad, 'Invariant varieties through singularities of holomorphic vector fields', Ann. of Math. (2) 115 (1982), 579-595.

[7] C. Camacho and B. Scárdua, 'Holomorphic foliations with Liouvillian first integrals', Ergod. Th. $\mathcal{E}$ Dynam. Sys. 21 (2001), 717-756. 
[8] H. Dulac, 'Solutions d'un système d'équations différentielles dans le voisinage de valeurs singulières', Bull. Soc. Math. France 40 (1912), 324-383.

[9] C. Godbillon, Feuilletages. Etudes Géométriques, Progress in Mathematics, 98 (Birkhäuser, Basel, 1991).

[10] R. C. Gunning, Introduction to Holomorphic Functions of Several Variables. Vol. I. Function Theory, The Wadsworth \& Brooks/Cole Mathematics Series (Wadsworth \& Brooks/Cole, Pacific Grove, CA, 1990).

[11] R. C. Gunning, Introduction to Holomorphic Functions of Several Variables. Vol. II. Local Theory, The Wadsworth \& Brooks/Cole Mathematics Series (Wadsworth \& Brooks/Cole, Pacific Grove, CA, 1990).

[12] R. C. Gunning and H. Rossi, Analytic Functions of Several Complex Variables (Prentice Hall, Englewood Cliffs, NJ, 1965).

[13] J. Martinet and J.-P. Ramis, 'Problème de modules pour des équations différentielles non linéaires du premier ordre', Publ. Math. Inst. Hautes Études Sci. 55 (1982), 63-124.

[14] J. Martinet and J.-P. Ramis, 'Classification analytique des équations différentielles non linéaires résonnants du premier ordre', Ann. Sci. Éc. Norm. Supér. (4) 16 (1983), 571-621.

[15] J-.F. Mattei and R. Moussu, 'Holonomie et intégrales premières', Ann. Sci. Éc. Norm. Supér. (4) 13 (1980), 469-523.

[16] B. Scárdua, 'Transversely affine and transversely projective holomorphic foliations', Ann. Sci. Éc. Norm. Supér. 30 (1997), 169-204.

[17] B. Scárdua, 'Integration of complex differential equations', J. Dyn. Control Syst. 5 (1999), 1-50.

[18] A. Seidenberg, 'Reduction of singularities of the differential equation $A d y=B d x$ ', Amer. J. Math. 90 (1968), 248-269.

[19] C. L. Siegel, 'Iteration of analytic functions', Ann. of Math. (2) 43 (1942), 607-612.

[20] Y. Siu, Techniques of Extension of Analytic Objects (Marcel Dekker, New York, 1974).

[21] F. Touzet, 'Sur les feuilletages holomorphes transversalement projectifs', Ann. Inst. Fourier (Grenoble) $\mathbf{5 3}$ (2003), 815-846.

ALBETÃ MAFRA, Instituto de Matemática, Universidade Federal do Rio de Janeiro, Caixa Postal 68530, 21945-970-Rio de Janeiro, Brazil e-mail: albetan@im.ufrj.br

BRUNO SCARDUA, Instituto de Matemática, Universidade Federal do Rio de Janeiro, Caixa Postal 68530, 21945-970-Rio de Janeiro, Brazil e-mail: scardua@im.ufrj.br 